\title{
Existence and Globally Asymptotic Stability of Equilibrium Solution for Fractional-Order Hybrid BAM Neural Networks with Distributed Delays and Impulses
}

\author{
Hai Zhang, ${ }^{1}$ Renyu Ye, ${ }^{1,2}$ Jinde Cao, ${ }^{3,4}$ and Ahmed Alsaedi ${ }^{5}$ \\ ${ }^{1}$ School of Mathematics and Computation Science, Anqing Normal University, Anqing 246133, China \\ ${ }^{2}$ College of Science, Nanjing University of Aeronautics and Astronautics, Nanjing 211106, China \\ ${ }^{3}$ School of Mathematics and Research Center for Complex Systems and Network Sciences, Southeast University, \\ Nanjing 210096, China \\ ${ }^{4}$ Department of Mathematics, Faculty of Science, King Abdulaziz University, Jeddah 21589, Saudi Arabia \\ ${ }^{5}$ Nonlinear Analysis and Applied Mathematics (NAAM) Research Group, Department of Mathematics, \\ King Abdulaziz University, Jeddah 21589, Saudi Arabia \\ Correspondence should be addressed to Jinde Cao; jdcao@seu.edu.cn
}

Received 7 May 2017; Revised 10 July 2017; Accepted 20 July 2017; Published 13 September 2017

Academic Editor: Amr Elsonbaty

Copyright (C) 2017 Hai Zhang et al. This is an open access article distributed under the Creative Commons Attribution License, which permits unrestricted use, distribution, and reproduction in any medium, provided the original work is properly cited.

\begin{abstract}
This paper investigates the existence and globally asymptotic stability of equilibrium solution for Riemann-Liouville fractionalorder hybrid BAM neural networks with distributed delays and impulses. The factors of such network systems including the distributed delays, impulsive effects, and two different fractional-order derivatives between the $U$-layer and $V$-layer are taken into account synchronously. Based on the contraction mapping principle, the sufficient conditions are derived to ensure the existence and uniqueness of the equilibrium solution for such network systems. By constructing a novel Lyapunov functional composed of fractional integral and definite integral terms, the globally asymptotic stability criteria of the equilibrium solution are obtained, which are dependent on the order of fractional derivative and network parameters. The advantage of our constructed method is that one may directly calculate integer-order derivative of the Lyapunov functional. A numerical example is also presented to show the validity and feasibility of the theoretical results.
\end{abstract}

\section{Introduction}

Since fractional derivatives are nonlocal and have weakly singular kernels, the subject of fractional calculus has been attracting attention and interest in various fields of diffusion [1], physics [2], market dynamics [3], engineering [4], control system [5], biological system [6], financial system [7], epidemic model [8], and so on. At the same time, fractional-order differential equations have been proved to be an excellent tool in the modelling of many phenomena [9-11]. Recently, some important advances on dynamical behaviors such as chaos phenomena, Hopf bifurcation, synchronization control, and stabilization problems for fractional-order systems or fractional-order practical models have been reported in [12-16]. These proposed results show the superiority and importance of fractional calculus and effectively motivate the development of new applied fields.

Note that various classes of neural networks such as Hopfield neural networks $[17,18]$, recurrent neural networks $[19,20]$, cellular neural networks [21], Cohen-Grossberg neural networks [22], and bidirectional associative memory (BAM) neural networks [23-25] have been widely used in solving some signal processing, optimization, and image processing problems. In the last few years, some researchers have introduced fractional operators to neural networks to form fractional-order neural models [26-30], which could better describe the dynamical behaviors of the neurons. As an important dynamic behavior, stability is one of the most concerned problems for any dynamic system. For example, Song and Cao [26] have established some sufficient conditions to 
ensure the existence and uniqueness of the nontrivial solution by using the contraction mapping principle, Krasnoselskii fixed point theorem, and the inequality technique, in which uniform stability conditions of fractional-order neural networks are also derived in fixed time-intervals. Note that timedelay (see [23-25, 31-37]) is a common phenomenon and is inevitable in practice, which often exists in almost every neural network and has an important effect on the stability and performance of system.

There are also several recent results discussing the topics including stability analysis for fractional-order dynamical systems in $[38,39]$. For instance, the stability problems of main concern for control theory in finite-dimensional linear fractional-order systems have been considered [38], in which both internal and external stabilities for fractional-order differential systems in state-space form have been studied. For fractional-order differential systems in polynomial representation, the external stability has been thoroughly discussed. In [39], Matouk has investigated the stability conditions of a class of fractional-order hyperchaotic systems; then the stability conditions have been applied to a novel fractional-order hyperchaotic system. Based on the Routh-Hurwitz theorem, the conditions for controlling hyperchaos via feedback control approach have also been derived. At the same time, the various kinds of stability of delayed fractional-order neural networks have been extensively investigated. For example, Mittag-Leffler stability of fractional-order delayed neural networks has been investigated by applying fractional Lyapunov direct method $[28,30,32]$. The finite-time stability of Caputo fractional-order delayed neural networks has been studied by applying Gronwall's inequality approach and inequality scaling techniques $[33,34]$. The delay-independent stability criteria of Riemann-Liouville fractional-order neutral-type delayed neural networks have been proposed based on classical Lyapunov functional method [35]. The uniform stability and global stability of fractional neural networks with delay are considered based on the fractional calculus theory and analytical techniques [36]. Global $o\left(t^{-\alpha}\right)$ stability and global asymptotical periodicity for a class of fractionalorder complex-valued neural networks with time-varying delays are discussed by using the fractional Lyapunov method and a Leibniz rule for fractional differentiation [37].

Although most dynamical systems are analyzed in either the continuous-time or discrete-time domain, many real systems in physics, engineering, chemistry, biology, and information science may experience abrupt changes as certain instants during the continuous dynamical processes. This kind of impulsive behaviors can be modelled by impulsive systems [23, 25, 29, 32, 40-42]. On the other hand, bidirectional associative memory (BAM) neural networks attract many studies due to its extensive applications in many fields [22-25, 43-46]. In [43], Kosko first introduced hybrid BAM neural network models. The remarkable feature of the proposed BAM neural networks lies in the close relation of the neurons between the $U$-layer and $V$-layer. That is, the neurons in one layer are fully interconnected to the one in the other layer, but there are not any interconnections among neurons in the same layer. It is worth mentioning that many contributions have been made concerning the dynamics of fractional-order BAM delayed neural networks (see [4446]) including finite-time stability [44] and Mittag-Leffler synchronization [45]. In [46], globally asymptotic stability problem of impulsive fractional-order neural networks with discrete delays has been studied, yet the existence of the equilibrium solution for fractional-order BAM neural networks has not been taken into account. On the other hand, it should be pointed out that the finite-time stability and asymptotic stability in the sense of Lyapunov are different concepts, because finite-time stability does not contain Lyapunov asymptotic stability and vice versa [34, 47]. Although the signal transmission is sometimes instantaneous modelling with discrete delays, it may be sometimes a distribution propagation delay over a period of time so that distributed delays (see $[20,23,25]$ ) should not be ignored in the model. Compared to the advances of integer-order neural networks with or without time delays, the research on the stability of fractional-order BAM delayed neural networks is still at the stage of exploiting and developing [44-46]. To the best of our knowledge, there are few papers on investigating the global stability of the fractional-order hybrid BAM neural networks with both impulse and distributed delay in the current literature.

Motivated by the above discussions, this paper investigates the existence and globally asymptotic stability of equilibrium solution for impulsive Riemann-Liouville fractionalorder hybrid BAM neural networks with distributed delays. The factors of such network systems including the distributed delays, impulses, and two different fractional-order derivatives between the $U$-layer and $V$ - layer are taken into account synchronously. Based on the contraction mapping principle, the sufficient conditions are presented for the existence and uniqueness of the equilibrium solution for such network systems. By constructing a suitable Lyapunov functional associated with fractional integral terms, the globally asymptotic stability criteria of the equilibrium point are derived. The advantage of constructing the Lyapunov functional is that one can directly calculate its first-order derivative to check global stability. A numerical example is also given to show the validity and feasibility of the theoretical results.

This paper is organized as follows. In Section 2, we recall some definitions concerning fractional calculus and describe impulsive Riemann-Liouville fractional-order BAM neural networks with distributed delays. In Section 3, the existence and uniqueness of the equilibrium solution for such network systems are discussed based on the contraction mapping principle. In Section 4, the globally asymptotic stability criteria of the equilibrium solution are derived. An illustrative example is given to show the effectiveness and applicability of the proposed results in Section 5. Finally, some concluding remarks are drawn in Section 6.

\section{Preliminaries and Model Description}

In this section, we recall the definitions of fractional calculus and several basic lemmas. Moreover, we describe a class of impulsive fractional-order hybrid BAM neural network models with distributed delays. 
Definition 1 (see [10]). The Riemann-Liouville fractional integral of order $q$ for a function $f$ is defined as

$$
t_{0} D_{t}^{-q} f(t)=\frac{1}{\Gamma(q)} \int_{t_{0}}^{t}(t-s)^{q-1} f(s) d s,
$$

where $q>0, t \geqslant t_{0}$. The Gamma function $\Gamma(q)$ is defined by the integral

$$
\Gamma(z)=\int_{0}^{+\infty} s^{z-1} e^{-s} d s, \quad(\mathfrak{R e}(z)>0) .
$$

Currently, there exist several definitions about the fractional derivative of order $q>0$ including Grünwald-Letnikov (GL) definition, Riemann-Liouville (RL) definition, and Caputo definition [9-11]. In this paper, our consideration is the fractional-order neural networks with Riemann-Liouville derivative, whose definition and properties are given below.

Definition 2 (see [10]). The Riemann-Liouville fractional derivative of order $q$ for a function $f$ is defined as

$$
\begin{aligned}
{ }_{t_{0}}^{\mathrm{RL}} D_{t}^{q} f(t) & =\frac{d^{m}}{d t^{m}}\left[t_{0} D_{t}^{-(m-q)} f(t)\right] \\
& =\frac{1}{\Gamma(m-q)} \frac{d^{m}}{d t^{m}} \int_{t_{0}}^{t}(t-s)^{m-q-1} f(s) d s
\end{aligned}
$$

where $0 \leqslant m-1<q<m, m \in \mathbb{Z}^{+}$.

In particular, for $\alpha \in(0,1)$ case, the Riemann-Liouville fractional derivative of order $\alpha$ for a constant $x^{*}$ is

$$
{ }_{0}^{\mathrm{RL}} D_{t}^{\alpha} x^{*}=\frac{t^{-\alpha}}{\Gamma(1-\alpha)} x^{*} .
$$

Lemma 3 (see [10]). If $f(t), g(t) \in \mathbf{C}^{m}\left[t_{0}, b\right]$, and $m-1 \leqslant$ $p<m \in \mathbb{Z}^{+}$, then

(1) ${ }_{t_{0}}^{\mathrm{RL}} D_{t}^{q}\left(L_{1} f(t)+L_{2} g(t)\right)=L_{1}{ }_{t_{0}}^{\mathrm{RL}} D_{t}^{q} f(t)+L_{2}{ }_{t_{0}}^{\mathrm{RL}} D_{t}^{q} g(t)$, $L_{1}, t_{2} \in \mathbb{R}, q>0$

(2) ${ }_{t_{0}} D_{t}^{-p}\left({ }_{t_{0}} D_{t}^{-q} f(t)\right)={ }_{t_{0}} D_{t}^{-(p+q)} f(t), p, q>0$;

(3) ${ }_{t_{0}}^{\mathrm{RL}} D_{t}^{p}\left({ }_{t_{0}} D_{t}^{-q} f(t)\right)={ }_{t_{0}}^{\mathrm{RL}} D_{t}^{p-q} f(t), p>q>0$;

(4) ${ }_{t_{0}}^{\mathrm{RL}} D_{t}^{p}\left({ }_{t_{0}} D_{t}^{-q} f(t)\right)={ }_{t_{0}} D_{t}^{-(q-p)} f(t), q>p>0$.

The following lemmas will be used in the proof of our main results.

Lemma 4 (contraction mapping principle [48]). Suppose that $(X, \rho)$ is a complete metric space, $\Phi: X \rightarrow X$, and there is some real number $0<k<1$ such that

$$
\rho(\Phi(x), \Phi(y)) \leqslant k \rho(x, y), \quad \forall x, y \in X
$$

then there is a unique point $x_{0} \in X$ such that $\Phi\left(x_{0}\right)=x_{0}$.

Lemma 5 (fractional Barbalat lemma [42]). If $\int_{t_{0}}^{t} w(s) d s$ has a finite limit as $t \rightarrow+\infty$, and ${ }_{t_{0}}^{\mathrm{RL}} D_{t}^{\alpha} w(t)$ is bounded, then $w(t) \rightarrow$ 0 as $t \rightarrow+\infty$, where $0<\alpha<1$.
In this paper, we consider the Riemann-Liouville fractional-order hybrid BAM neural network models with distributed delay and impulsive effects described by the following states equations:

$$
\begin{aligned}
&{ }_{0}^{\mathrm{RL}} D_{t}^{\alpha} x_{i}(t)=-a_{i} x_{i}(t)+\sum_{j=1}^{m} b_{i j} f_{j}\left(y_{j}(t)\right) \\
&+\sum_{j=1}^{m} \int_{0}^{\tau} r_{i j}(s) f_{j}\left(y_{j}(t-s)\right) d s+I_{i}, \\
& t>0, t \neq t_{k},
\end{aligned}
$$

$$
\begin{aligned}
\Delta x_{i}\left(t_{k}\right)= & \gamma_{k}^{(1)}\left(x_{i}\left(t_{k}\right)\right), \\
& i=1,2, \ldots, n ; k=1,2, \ldots, \\
{ }_{0}^{\mathrm{RL}} D_{t}^{\beta} y_{j}(t)= & -c_{j} y_{j}(t)+\sum_{i=1}^{n} d_{j i} g_{i}\left(x_{i}(t)\right) \\
& +\sum_{i=1}^{n} \int_{0}^{\tau} p_{j i}(s) g_{i}\left(x_{i}(t-s)\right) d s+J_{j}, \\
& t>0, t \neq t_{k},
\end{aligned}
$$

$$
\Delta y_{j}\left(t_{k}\right)=\gamma_{k}^{(2)}\left(y_{j}\left(t_{k}\right)\right)
$$

$$
j=1,2, \ldots, m ; k=1,2, \ldots,
$$

where $U=\left\{x_{1}, x_{2}, \ldots, x_{n}\right\}$ and $V=\left\{y_{1}, y_{2}, \ldots, y_{m}\right\}$ are two layers in the BAM model $(6) ; x_{i}(t)$ and $y_{j}(t)$ are state variables of $i$ th neuron in the $U$-layer and $j$ th neuron in the $V$-layer, respectively; ${ }_{0}^{\mathrm{RL}} D_{t}^{\alpha} x_{i}(\cdot)$ and ${ }_{0}^{\mathrm{RL}} D_{t}^{\beta} y_{j}(\cdot)$ denote an $\alpha$ and a $\beta$ order Riemann-Liouville fractional-order derivative of $x_{i}(\cdot)$ and $y_{j}(\cdot)$, respectively; the constants $\alpha$ and $\beta$ satisfy $0<\alpha<$ $1,0<\beta<1 . a_{i}>0$ and $c_{j}>0$ denote decay coefficients of signals from neurons $x_{i}$ to $y_{j}$, respectively; $f_{i}$ and $g_{j}$ are the neuron activation functions; $b_{i j}, d_{j i}, r_{i j}(t)$ and $p_{j i}(t)$ represent the weight coefficients of the neurons; $I_{i}$ and $J_{j}$ denote the external inputs of $U$-layer and $V$-layer, respectively; $\tau>$ 0 denotes the maximum possible transmission delay from neuron to another. Moreover, impulsive moments $\left\{t_{k} \mid k=\right.$ $1,2, \ldots\}$ satisfy $0=t_{0}<t_{1}<t_{2}<\cdots<t_{k}<\cdots, t_{k} \rightarrow+\infty$ as $k \rightarrow+\infty$, and

$$
\begin{aligned}
\Delta x_{i}\left(t_{k}\right)= & x_{i}\left(t_{k}^{+}\right)-x_{i}\left(t_{k}^{-}\right), \\
& x_{i}\left(t_{k}^{+}\right)=\lim _{\varepsilon \rightarrow 0^{+}} x_{i}\left(t_{k}+\varepsilon\right), x_{i}\left(t_{k}^{-}\right)=x_{i}\left(t_{k}\right), \\
\Delta y_{j}\left(t_{k}\right)= & y_{j}\left(t_{k}^{+}\right)-y_{j}\left(t_{k}^{-}\right), \\
& y_{j}\left(t_{k}^{+}\right)=\lim _{\varepsilon \rightarrow 0^{+}} y_{j}\left(t_{k}+\varepsilon\right), y_{j}\left(t_{k}^{-}\right)=y_{j}\left(t_{k}\right),
\end{aligned}
$$

where $x_{i}\left(t_{k}^{+}\right)$and $x_{i}\left(t_{k}^{-}\right)$represent the right and left limits of $x_{i}(t)$ at $t=t_{k}$, respectively; $x_{i}\left(t_{k}^{-}\right)=x_{i}\left(t_{k}\right)$ and $y_{j}\left(t_{k}^{-}\right)=y_{j}\left(t_{k}\right)$ imply that $x_{i}(t)$ and $y_{j}(t)$ are both left continuous at $t=$ $t_{k}$. The initial conditions associated with Riemann-Liouville 
fractional-order network system (6) can be expressed as (see [9-11])

$$
\begin{aligned}
{ }_{0} D_{t}^{-(1-\alpha)} x_{i}(t) & =\varphi_{i}(t), \\
{ }_{0} D_{t}^{-(1-\alpha)} y_{j}(t) & =\psi_{j}(t), \\
i= & 1,2, \ldots, n ; j=1,2, \ldots, m, t \in[-\tau, 0] .
\end{aligned}
$$

Throughout this paper, we assume that the neuron activation functions $f_{j}, g_{i}$ and impulsive operators $\gamma_{k}^{(1)}\left(x_{i}\left(t_{k}\right)\right)$, $\gamma_{k}^{(2)}\left(y_{j}\left(t_{k}\right)\right)$ satisfy the following conditions:

(H1) For $i=1,2, \ldots, n ; j=1,2, \ldots, m$, the functions $r_{i j}(\cdot)$ and $p_{j i}(\cdot)$ are continuous on $[0, \tau]$. Thus, there exist positive constants $R_{i j}, P_{j i} \in \mathbb{R}^{+}$such that

$$
\begin{gathered}
\left|r_{i j}(s)\right| \leqslant R_{i j}, \\
\left|p_{j i}(s)\right| \leqslant P_{j i},
\end{gathered}
$$

$\forall s \in[0, \tau]$.

(H2) The neuron activation functions $f_{j}(\cdot), g_{i}(\cdot)(i=$ $1,2, \ldots, n ; j=1,2, \ldots, m)$ are Lipschitz continuous. That is, there exist positive constants $F_{j}, G_{j} \in \mathbb{R}^{+}$such that

$$
\begin{gathered}
\left|f_{j}(x)-f_{j}(y)\right| \leqslant F_{j}|x-y|, \\
\left|g_{i}(x)-g_{i}(y)\right| \leqslant G_{i}|x-y|,
\end{gathered}
$$

\section{$\forall x, y \in \mathbb{R}$.} satisfy

(H3) The impulsive operators $\gamma_{k}^{(1)}\left(x_{i}\left(t_{k}\right)\right)$ and $\gamma_{k}^{(2)}\left(y_{j}\left(t_{k}\right)\right)$

$$
\begin{gathered}
\gamma_{k}^{(1)}\left(x_{i}\left(t_{k}\right)\right)=-\lambda_{i k}^{(1)}\left(x_{i}\left(t_{k}\right)-x_{i}^{*}\right), \\
i=1,2, \ldots, n ; k=1,2, \ldots, \\
\gamma_{k}^{(2)}\left(y_{j}\left(t_{k}\right)\right)=-\lambda_{j k}^{(2)}\left(y_{j}\left(t_{k}\right)-y_{j}^{*}\right), \\
j=1,2, \ldots, m ; k=1,2, \ldots,
\end{gathered}
$$

where $\lambda_{i k}^{(1)} \in(0,2)(i=1,2, \ldots, n ; k=1,2, \ldots)$, and $\lambda_{j k}^{(2)} \in$ $(0,2)(j=1,2, \ldots, m ; k=1,2, \ldots)$.

Remark 6. The purpose of this paper is to investigate the existence and globally asymptotic stability conditions of the equilibrium solution for fractional-order BAM network model (6). In discussing the stability of neural networks, the neuron activation functions are usually assumed to be bounded, monotonic [23], and differential [36, 37]. In system (6), the neuron activation functions are not necessarily bounded, monotonic, and differential. Therefore, the globally asymptotic stability criteria are more general and less conservative in this paper.

\section{Existence of Equilibrium Solution}

In this section, the sufficient conditions for the existence and uniqueness of the equilibrium solution of system (6) are derived based on the contraction mapping principle [48].
Similar to integer-order differential systems, we first define the equilibrium solution of fractional-order network systems. It should be pointed out that Riemann-Liouville fractional-order derivative of a nonzero constant is not equal to zero, which leads to the remarkable difference of the equilibrium solution between integer-order systems and Riemann-Liouville fractional-order systems.

Definition 7. A constant vector $\left(x^{* T}, y^{* T}\right)^{T}=\left(x_{1}^{*}, x_{2}^{*}, \ldots, x_{n}^{*}\right.$, $\left.y_{1}^{*}, y_{2}^{*}, \ldots, y_{m}^{*}\right)^{T} \in \mathbb{R}^{n+m}$ is an equilibrium solution of system (6) if and only if $x^{*}=\left(x_{1}^{*}, x_{2}^{*}, \ldots, x_{n}^{*}\right)^{T}$ and $y^{*}=\left(y_{1}^{*}, y_{2}^{*}, \ldots\right.$, $\left.y_{m}^{*}\right)^{T}$ satisfy the following equations:

$$
\begin{aligned}
{ }_{0}^{\mathrm{RL}} D_{t}^{\alpha}\left\{x_{i}^{*}\right\}= & -a_{i} x_{i}^{*}+\sum_{j=1}^{m} b_{i j} f_{j}\left(y_{j}^{*}\right) \\
& +\sum_{j=1}^{m} \int_{0}^{\tau} r_{i j}(s) f_{j}\left(y_{j}^{*}\right) d s+I_{i},
\end{aligned}
$$

$$
i=1,2, \ldots, n \text {, }
$$

$$
\begin{aligned}
{ }_{0}^{\mathrm{RL}} D_{t}^{\beta}\left\{y_{j}^{*}\right\}= & -c_{j} y_{j}^{*}+\sum_{i=1}^{n} d_{j i} g_{i}\left(x_{i}^{*}\right) \\
& +\sum_{i=1}^{n} \int_{0}^{\tau} p_{j i}(s) g_{i}\left(x_{i}^{*}\right) d s+J_{j},
\end{aligned}
$$$$
j=1,2, \ldots, m
$$

and the impulsive jumps $\gamma_{k}^{(1)}\left(x_{i}\left(t_{k}\right)\right)$ and $\gamma_{k}^{(2)}\left(y_{j}\left(t_{k}\right)\right)$ are assumed to satisfy

$$
\begin{aligned}
\gamma_{k}^{(1)}\left(x_{i}^{*}\right) & =0, \\
\gamma_{k}^{(2)}\left(y_{j}^{*}\right) & =0, \\
i & =1,2, \ldots, n ; j=1,2, \ldots, m ; k=1,2, \ldots
\end{aligned}
$$
$\mathbb{R}^{n+m}$

In what follows, we use the following vector norm of

$$
\|u\|=\sum_{i=1}^{n+m}\left|u_{i}\right|, \quad u=\left(u_{1}, u_{2}, \ldots, u_{n+m}\right)^{T} \in \mathbb{R}^{n+m}
$$

Theorem 8. Suppose that conditions (H1)-(H3) hold; then there exists a unique equilibrium solution for system (6), if the following inequalities simultaneously hold for a small enough constant $\varepsilon>0$

$$
\begin{aligned}
\omega_{1} & =\max _{1 \leqslant i \leqslant n}\left\{\frac{\varepsilon}{\Gamma(1-\alpha)} \cdot \frac{1}{a_{i}}+\frac{G_{i}}{a_{i}} \sum_{j=1}^{m}\left[\left|d_{j i}\right|+\tau P_{j i}\right]\right\} \\
& <1,
\end{aligned}
$$




$$
\begin{aligned}
\omega_{2} & =\max _{1 \leqslant j \leqslant m}\left\{\frac{\varepsilon}{\Gamma(1-\beta)} \cdot \frac{1}{c_{j}}+\frac{F_{j}}{c_{j}} \sum_{i=1}^{n}\left[\left|b_{i j}\right|+\tau R_{i j}\right]\right\} \\
& <1 .
\end{aligned}
$$

Proof. According to Definition 2, for $\alpha, \beta \in(0,1)$, the Riemann-Liouville fractional-order derivatives of the constants $u_{i}^{*}$ and $v_{j}^{*}$ can be written as the following forms:

$$
\begin{aligned}
& { }_{0}^{\mathrm{RL}} D_{t}^{\alpha} u_{i}^{*}=\frac{t^{-\alpha}}{\Gamma(1-\alpha)} u_{i}^{*}, \\
& { }_{0}^{\mathrm{RL}} D_{t}^{\beta} v_{j}^{*}=\frac{t^{-\beta}}{\Gamma(1-\beta)} v_{j}^{*},
\end{aligned}
$$

Define a mapping $\Phi: \mathbb{R}^{n+m} \rightarrow \mathbb{R}^{n+m}$, where $\mathbf{u}=\left(u_{1}, \ldots, u_{n}\right.$, $\left.v_{1}, \ldots, v_{m}\right)^{T} \in \mathbb{R}^{n+m}$ and

$$
\Phi(\mathbf{u})=\left[\begin{array}{c}
\sum_{j=1}^{m} b_{1 j} f_{j}\left(\frac{v_{j}}{c_{j}}\right)+\sum_{j=1}^{m} \int_{0}^{\tau} r_{1 j}(s) f_{j}\left(\frac{v_{j}}{c_{j}}\right) d s+I_{1}-\frac{t^{-\alpha}}{\Gamma(1-\alpha)} \frac{u_{1}}{a_{1}} \\
\vdots \\
\sum_{j=1}^{m} b_{n j} f_{j}\left(\frac{v_{j}}{c_{j}}\right)+\sum_{j=1}^{m} \int_{0}^{\tau} r_{n j}(s) f_{j}\left(\frac{v_{j}}{c_{j}}\right) d s+I_{n}-\frac{t^{-\alpha}}{\Gamma(1-\alpha)} \frac{u_{n}}{a_{n}} \\
\sum_{i=1}^{n} d_{1 i} g_{i}\left(\frac{u_{i}}{a_{i}}\right)+\sum_{i=1}^{n} \int_{0}^{\tau} p_{1 i}(s) g_{i}\left(\frac{u_{i}}{a_{i}}\right) d s+J_{1}-\frac{t^{-\beta}}{\Gamma(1-\beta)} \frac{v_{1}}{c_{1}} \\
\vdots \\
\sum_{i=1}^{n} d_{m i} g_{i}\left(\frac{u_{i}}{a_{i}}\right)+\sum_{i=1}^{n} \int_{0}^{\tau} p_{m i}(s) g_{i}\left(\frac{u_{i}}{a_{i}}\right) d s+J_{m}-\frac{t^{-\beta}}{\Gamma(1-\beta)} \frac{v_{\mathrm{m}}}{c_{m}}
\end{array}\right] .
$$

Consider $\forall \overline{\mathbf{u}}=\left(\bar{u}_{1}, \ldots, \bar{u}_{n}, \bar{v}_{1}, \ldots, \bar{v}_{m}\right)^{T} \in \mathbb{R}^{n+m}$; then it follows from (14) that

$$
\begin{aligned}
\|\Phi(\mathbf{u})-\Phi(\overline{\mathbf{u}})\| \leqslant & \sum_{i=1}^{n}\left|\sum_{j=1}^{m}\left\{b_{i j}\left[f_{j}\left(\frac{v_{j}}{c_{j}}\right)-f_{j}\left(\frac{\bar{v}_{j}}{c_{j}}\right)\right]+\int_{0}^{\tau} r_{i j}(s)\left[f_{j}\left(\frac{v_{j}}{c_{j}}\right)-f_{j}\left(\frac{\bar{v}_{j}}{c_{j}}\right)\right] d s\right\}\right| \\
& +\sum_{j=1}^{m}\left|\sum_{i=1}^{n}\left\{d_{j i}\left[g_{i}\left(\frac{u_{i}}{a_{i}}\right)-g_{i}\left(\frac{\bar{u}_{i}}{a_{i}}\right)\right]+\int_{0}^{\tau} p_{j i}(s)\left[g_{i}\left(\frac{u_{i}}{a_{i}}\right)-g_{i}\left(\frac{\bar{u}_{i}}{a_{i}}\right)\right] d s\right\}\right| \\
& +\sum_{i=1}^{n}\left|\frac{t^{-\alpha}}{\Gamma(1-\alpha)}\left[\frac{u_{i}}{a_{i}}-\frac{\bar{u}_{i}}{a_{i}}\right]\right|+\sum_{j=1}^{m}\left|\frac{t^{-\beta}}{\Gamma(1-\beta)}\left[\frac{v_{j}}{c_{j}}-\frac{\bar{v}_{j}}{c_{j}}\right]\right| .
\end{aligned}
$$

According to (H1)-(H2), one has

$$
\begin{gathered}
\|\Phi(\mathbf{u})-\Phi(\overline{\mathbf{u}})\| \leqslant \sum_{i=1}^{n} \sum_{j=1}^{m}\left\{\left|b_{i j}\right| F_{j}\left|\frac{v_{j}-\bar{v}_{j}}{c_{j}}\right|\right. \\
\left.+\int_{0}^{\tau}\left|r_{i j}(s)\right| F_{j}\left|\frac{v_{j}-\bar{v}_{j}}{c_{j}}\right| d s\right\} \\
+\sum_{j=1}^{m} \sum_{i=1}^{n}\left\{\left|d_{j i}\right| G_{i}\left|\frac{u_{i}-\bar{u}_{i}}{a_{i}}\right|\right. \\
\left.+\int_{0}^{\tau}\left|p_{j i}(s)\right| G_{i}\left|\frac{u_{i}-\bar{u}_{i}}{a_{i}}\right| d s\right\}+\frac{t^{-\alpha}}{\Gamma(1-\alpha)}
\end{gathered}
$$

$$
\begin{aligned}
& \cdot \max _{1 \leqslant i \leqslant n}\left\{\frac{1}{a_{i}}\right\} \cdot \sum_{i=1}^{n}\left|u_{i}-\bar{u}_{i}\right|+\frac{t^{-\beta}}{\Gamma(1-\beta)} \cdot \max _{1 \leqslant j \leqslant m}\left\{\frac{1}{c_{j}}\right\} \\
& \cdot \sum_{j=1}^{m}\left|v_{j}-\bar{v}_{j}\right|,
\end{aligned}
$$

For $\alpha, \beta \in(0,1)$, we have $\lim _{t \rightarrow+\infty} t^{-\alpha}=0, \lim _{t \rightarrow+\infty} t^{-\beta}=0$. Therefore, there exists a small enough constant $\varepsilon>0$ such that $t^{-\alpha}<\varepsilon, t^{-\beta}<\varepsilon$. Thus, it follows from (19) that

$$
\begin{gathered}
\|\Phi(\mathbf{u})-\Phi(\overline{\mathbf{u}})\| \leqslant \sum_{i=1}^{n} \sum_{j=1}^{m}\left[\frac{\left|b_{i j}\right|+\tau R_{i j}}{c_{j}} F_{j}\left|v_{j}-\bar{v}_{j}\right|\right] \\
+\sum_{j=1}^{m} \sum_{i=1}^{n}\left[\frac{\left|d_{j i}\right|+\tau P_{j i}}{a_{i}} G_{i}\left|u_{i}-\bar{u}_{i}\right|\right]+\frac{\varepsilon}{\Gamma(1-\alpha)}
\end{gathered}
$$




$$
\begin{aligned}
& \cdot \max _{1 \leqslant i \leqslant n}\left\{\frac{1}{a_{i}}\right\} \cdot \sum_{i=1}^{n}\left|u_{i}-\bar{u}_{i}\right|+\frac{\varepsilon}{\Gamma(1-\beta)} \cdot \max _{1 \leqslant j \leqslant m}\left\{\frac{1}{c_{j}}\right\} \\
& \cdot \sum_{j=1}^{m}\left|v_{j}-\bar{v}_{j}\right| \\
& \leqslant \max _{1 \leqslant j \leqslant m}\left\{\frac{\varepsilon}{\Gamma(1-\beta)} \cdot \frac{1}{c_{j}}+\frac{F_{j}}{c_{j}} \sum_{i=1}^{n}\left[\left|b_{i j}\right|+\tau R_{i j}\right]\right\} \\
& \cdot \sum_{j=1}^{m}\left|v_{j}-\bar{v}_{j}\right| \\
& +\max _{1 \leqslant i \leqslant n}\left\{\frac{\varepsilon}{\Gamma(1-\alpha)} \cdot \frac{1}{a_{i}}+\frac{G_{i}}{a_{i}} \sum_{j=1}^{m}\left[\left|d_{j i}\right|+\tau P_{j i}\right]\right\} \\
& \cdot \sum_{i=1}^{n}\left|u_{i}-\bar{u}_{i}\right| \cdot
\end{aligned}
$$

Let $k=\max \left\{\omega_{1}, \omega_{2}\right\}$, where $\omega_{1}$ and $\omega_{2}$ are defined in (15). Hence, we have

$$
\begin{aligned}
\|\Phi(\mathbf{u})-\Phi(\overline{\mathbf{u}})\| & \leqslant k\left[\sum_{i=1}^{n}\left|u_{i}-\bar{u}_{i}\right|+\sum_{j=1}^{m}\left|v_{j}-\bar{v}_{j}\right|\right] \\
& =k\|\mathbf{u}-\overline{\mathbf{u}}\| .
\end{aligned}
$$

Thus, it follows from (15) that $0<k<1$, which implies that $\Phi: \mathbb{R}^{n+m} \rightarrow \mathbb{R}^{n+m}$ is a contraction mapping. Therefore, from Lemma 4 , there exists a unique fixed point of the map $\Phi$ : $\mathbb{R}^{n+m} \rightarrow \mathbb{R}^{n+m}$, such that $\Phi\left(\mathbf{u}^{*}\right)=\mathbf{u}^{*}$. Thus, from (17), we get

$$
\begin{gathered}
\sum_{j=1}^{m} b_{i j} f_{j}\left(\frac{v_{j}^{*}}{c_{j}}\right)+\sum_{j=1}^{m} \int_{0}^{\tau} r_{i j}(s) f_{j}\left(\frac{v_{j}^{*}}{c_{j}}\right) d s+I_{i} \\
-\frac{t^{-\alpha}}{\Gamma(1-\alpha)} \frac{u_{i}^{*}}{a_{i}}=u_{i}^{*}, \quad i=1,2, \ldots, n, \\
\sum_{i=1}^{n} d_{j i} g_{i}\left(\frac{u_{i}^{*}}{a_{i}}\right)+\sum_{i=1}^{n} \int_{0}^{\tau} p_{j i}(s) g_{i}\left(\frac{u_{i}^{*}}{a_{i}}\right) d s+J_{j} \\
-\frac{t^{-\beta}}{\Gamma(1-\beta)} \frac{v_{j}^{*}}{c_{j}}=v_{j}^{*}, \quad j=1,2, \ldots, m .
\end{gathered}
$$

Let $x_{i}^{*}=u_{i}^{*} / a_{i}, y_{j}^{*}=v_{j}^{*} / c_{j}$; then it follows from (22) that

$$
\begin{gathered}
\sum_{j=1}^{m} b_{i j} f_{j}\left(y_{j}^{*}\right)+\sum_{j=1}^{m} \int_{0}^{\tau} r_{i j}(s) f_{j}\left(y_{j}^{*}\right) d s+I_{i} \\
-\frac{t^{-\alpha}}{\Gamma(1-\alpha)} x_{i}^{*}=a_{i} x_{i}^{*}, \quad i=1,2, \ldots, n, \\
\sum_{i=1}^{n} d_{j i} g_{i}\left(x_{i}^{*}\right)+\sum_{i=1}^{n} \int_{0}^{\tau} p_{j i}(s) g_{i}\left(x_{i}^{*}\right) d s+J_{j} \\
-\frac{t^{-\beta}}{\Gamma(1-\beta)} y_{j}^{*}=c_{j} y_{j}^{*}, \quad j=1,2, \ldots, m ;
\end{gathered}
$$

that is

$$
\begin{aligned}
& \sum_{j=1}^{m} b_{i j} f_{j}\left(y_{j}^{*}\right)+\sum_{j=1}^{m} \int_{0}^{\tau} r_{i j}(s) f_{j}\left(y_{j}^{*}\right) d s+I_{i}-a_{i} x_{i}^{*} \\
& ={ }_{0}^{\mathrm{RL}} D_{t}^{\alpha}\left\{x_{i}^{*}\right\}, \quad i=1,2, \ldots, n, \\
& \sum_{i=1}^{n} d_{j i} g_{i}\left(x_{i}^{*}\right)+\sum_{i=1}^{n} \int_{0}^{\tau} p_{j i}(s) g_{i}\left(x_{i}^{*}\right) d s+J_{j}-c_{j} y_{j}^{*} \\
& ={ }_{0}^{\mathrm{RL}} D_{t}^{\beta}\left\{y_{j}^{*}\right\}, \quad j=1,2, \ldots, m .
\end{aligned}
$$

According to (H3), we know that

$$
\begin{aligned}
\gamma_{k}^{(1)}\left(x_{i}^{*}\right) & =0, \\
\gamma_{k}^{(2)}\left(y_{j}^{*}\right) & =0, \\
i & =1,2, \ldots, n ; j=1,2, \ldots, m ; k=1,2, \ldots
\end{aligned}
$$

Thus, it follows from Definition 7 that $\left(x_{1}^{*}, x_{2}^{*}, \ldots, x_{n}^{*}, y_{1}^{*}\right.$, $\left.y_{2}^{*}, \ldots, y_{m}^{*}\right)^{T} \in \mathbb{R}^{n+m}$ is a unique equilibrium solution for system (6). The proof is complete.

The following corollary is the direct result of Theorem 8 .

Corollary 9. Suppose that conditions (H1)-(H3) hold; then there exists a unique equilibrium solution for system (6), if the following inequalities simultaneously hold for a small enough constant $\varepsilon>0$

$$
\begin{aligned}
& \min _{1 \leqslant i \leqslant n}\left\{a_{i}-\frac{\varepsilon}{\Gamma(1-\alpha)}-G_{i} \sum_{j=1}^{m}\left[\left|d_{j i}\right|+\tau P_{j i}\right]\right\}>0, \\
& \min _{1 \leqslant j \leqslant m}\left\{c_{j}-\frac{\varepsilon}{\Gamma(1-\beta)}-F_{j} \sum_{i=1}^{n}\left[\left|b_{i j}\right|+\tau R_{i j}\right]\right\}>0 .
\end{aligned}
$$

Remark 10. Theorem 8 and Corollary 9 reveal that the conditions of existence and uniqueness of the equilibrium solution for system (6) are based on the contraction mapping principle, which can be expressed in terms of the algebraic inequalities. The conditions of existence and uniqueness of the equilibrium point for system (6) reflect the close relation between the coefficients, neuron activation functions, and time-delay of network parameters, which are also dependent on the orders $\alpha$ and $\beta$ of Riemann-Liouville derivatives. On the other hand, if we only assume that $(\mathbf{H 1})-(\mathbf{H} 3)$ hold, then there exists at least an equilibrium solution for system (6) by applying Schauder fixed point theorem, whose proof is omitted here.

\section{Globally Asymptotic Stability Criteria}

In this section, by constructing a novel Lyapunov functional, we obtain the sufficient conditions to ensure the globally asymptotic stability of the equilibrium solution for system (6) based on fractional Barbalat theorem and classical Lyapunov stability theory. 
Theorem 11. Suppose that conditions (H1)-(H3) hold; then a unique equilibrium solution for system (6) is globally asymptotically stable, if the following inequalities simultaneously hold for a small enough constant $\varepsilon>0$

$$
\begin{aligned}
& \eta_{1}=\min _{1 \leqslant i \leqslant n}\left\{a_{i}-G_{i} \sum_{j=1}^{m}\left[\left|d_{j i}\right|+\tau P_{j i}\right]\right\}>\frac{\varepsilon}{\Gamma(1-\alpha)}, \\
& \eta_{2}=\min _{1 \leqslant j \leqslant m}\left\{c_{j}-F_{j} \sum_{i=1}^{n}\left[\left|b_{i j}\right|+\tau R_{i j}\right]\right\}>\frac{\varepsilon}{\Gamma(1-\beta)} .
\end{aligned}
$$

Proof. From Corollary 9, there exists a unique equilibrium solution $\left(x^{* T}, y^{* T}\right)^{T}$ for system (6). By using the variable transformation method, we can shift the equilibrium point to the origin. Let $u_{i}(t)=x_{i}(t)-x_{i}^{*}, v_{j}(t)=y_{j}(t)-y_{j}^{*}$; then system (6) is transformed into

$$
\begin{gathered}
{ }_{0}^{\mathrm{RL}} D_{t}^{\alpha} u_{i}(t) \\
=-a_{i} u_{i}(t)+\sum_{j=1}^{m} b_{i j}\left[f_{j}\left(y_{j}(t)\right)-f_{j}\left(y_{j}^{*}\right)\right] \\
\quad+\sum_{j=1}^{m} \int_{0}^{\tau} r_{i j}(s)\left[f_{j}\left(y_{j}(t-s)\right)-f_{j}\left(y_{j}^{*}\right)\right] d s, \\
u_{i}\left(t_{k}^{+}\right)=\left(1-\lambda_{i k}^{(1)}\right) u_{i}\left(t_{k}^{-}\right), \\
\quad i=1,2, \ldots, n ; k=1,2, \ldots, \\
\mathrm{RL}_{0} D_{t}^{\beta} v_{j}(t) \\
=-c_{j} v_{j}(t)+\sum_{i=1}^{n} d_{j i}\left[g_{i}\left(x_{i}(t)\right)-g_{i}\left(x_{i}^{*}\right)\right] \\
+\sum_{i=1}^{n} \int_{0}^{\tau} p_{j i}(s)\left[g_{i} x_{i}(t-s)-g_{i}\left(x_{i}^{*}\right)\right] d s, \\
v_{j}\left(t_{k}^{+}\right)=\left(1-\lambda_{j k}^{(2)}\right) v_{j}\left(t_{k}^{-}\right), \\
j=1,2, \ldots, m ; k=1,2, \ldots
\end{gathered}
$$

Construct a novel Lyapunov functional composed of fractional-order integral and definite integral terms:

$$
\begin{aligned}
V(t)= & { }_{0} D_{t}^{-(1-\alpha)}\left[\sum_{i=1}^{n}\left|u_{i}(t)\right|\right] \\
& +{ }_{0} D_{t}^{-(1-\beta)}\left[\sum_{j=1}^{m}\left|v_{j}(t)\right|\right]
\end{aligned}
$$

$$
\begin{aligned}
& +\sum_{i=1}^{n} \sum_{j=1}^{m} F_{j}\left|b_{i j}\right| \int_{t-\tau}^{t}\left|v_{j}(s)\right| d s \\
& +\sum_{j=1}^{m} \sum_{i=1}^{n} G_{i}\left|d_{j i}\right| \int_{t-\tau}^{t}\left|u_{i}(s)\right| d s \\
& +\sum_{i=1}^{n} \sum_{j=1}^{m} F_{j} R_{i j} \int_{0}^{\tau} \int_{t-s}^{t}\left|v_{j}(\eta)\right| d \eta d s \\
& +\sum_{j=1}^{m} \sum_{i=1}^{n} G_{i} P_{j i} \int_{0}^{\tau} \int_{t-s}^{t}\left|u_{i}(\eta)\right| d \eta d s .
\end{aligned}
$$

The time derivative of $V(t)$ along the trajectories of system (6) can be calculated, which are carried out for the following cases.

Case 1. For $t \neq t_{k}$, from Lemma 3, we obtain

$$
\begin{aligned}
\frac{d^{+} V(t)}{d t}= & { }_{0}{ }_{0} D_{t}^{\alpha}\left[\sum_{i=1}^{n}\left|u_{i}(t)\right|\right]+{ }_{0}^{\mathrm{RL}} D_{t}^{\beta}\left[\sum_{j=1}^{m}\left|v_{j}(t)\right|\right] \\
& +\sum_{i=1}^{n} \sum_{j=1}^{m} F_{j}\left|b_{i j}\right|\left[\left|v_{j}(t)\right|-\left|v_{j}(t-\tau)\right|\right] \\
& +\sum_{j=1}^{m} \sum_{i=1}^{n} G_{i}\left|d_{j i}\right|\left[\left|u_{i}(t)\right|-\left|u_{i}(t-\tau)\right|\right] \\
& +\sum_{i=1}^{n} \sum_{j=1}^{m} F_{j} R_{i j} \int_{0}^{\tau}\left[\left|v_{j}(t)\right|-\left|v_{j}(t-s)\right|\right] d s \\
& +\sum_{j=1}^{m} \sum_{i=1}^{n} G_{i} P_{j i} \int_{0}^{\tau}\left[\left|u_{i}(t)\right|-\left|u_{i}(t-s)\right|\right] d s .
\end{aligned}
$$

An application of Definition 2 yields

$$
\begin{aligned}
& { }_{0}^{\mathrm{RL}} D_{t}^{\alpha}\left|u_{i}(t)\right|=\operatorname{sgn}\left(u_{i}(t)\right) \cdot\left({ }_{0}^{\mathrm{RL}} D_{t}^{\alpha} u_{i}(t)\right), \\
& { }_{0}^{\mathrm{RL}} D_{t}^{\beta}\left|v_{j}(t)\right|=\operatorname{sgn}\left(v_{j}(t)\right) \cdot\left({ }_{0}^{\mathrm{RL}} D_{t}^{\beta} v_{j}(t)\right),
\end{aligned}
$$

where $\operatorname{sgn}(\cdot)$ denotes the standard signum function. Thus, (30) can be rewritten as

$$
\begin{aligned}
\frac{d^{+} V(t)}{d t}= & \sum_{i=1}^{n} \operatorname{sgn}\left(u_{i}(t)\right)\left[{ }_{0}^{\mathrm{RL}} D_{t}^{\alpha}\left(u_{i}(t)\right)\right] \\
& +\sum_{j=1}^{m} \operatorname{sgn}\left(v_{j}(t)\right)\left[{ }_{0}^{\mathrm{RL}} D_{t}^{\beta}\left(v_{j}(t)\right)\right] \\
& +\sum_{i=1}^{n} \sum_{j=1}^{m} F_{j}\left|b_{i j}\right|\left[\left|v_{j}(t)\right|-\left|v_{j}(t-\tau)\right|\right]
\end{aligned}
$$




$$
\begin{aligned}
& +\sum_{j=1}^{m} \sum_{i=1}^{n} G_{i}\left|d_{j i}\right|\left[\left|u_{i}(t)\right|-\left|u_{i}(t-\tau)\right|\right] \\
& +\sum_{i=1}^{n} \sum_{j=1}^{m} F_{j} R_{i j} \int_{0}^{\tau}\left[\left|v_{j}(t)\right|-\left|v_{j}(t-s)\right|\right] d s \\
& +\sum_{j=1}^{m} \sum_{i=1}^{n} G_{i} P_{j i} \int_{0}^{\tau}\left[\left|u_{i}(t)\right|-\left|u_{i}(t-s)\right|\right] d s .
\end{aligned}
$$

Combining (28) and (32) yields

$$
\begin{aligned}
& \frac{d^{+} V(t)}{d t}=\sum_{i=1}^{n} \operatorname{sgn}\left(u_{i}(t)\right)\left\{-a_{i} u_{i}(t)\right. \\
& +\sum_{j=1}^{m} b_{i j}\left[f_{j}\left(y_{j}(t)\right)-f_{j}\left(y_{j}^{*}\right)\right] \\
& \left.+\sum_{j=1}^{m} \int_{0}^{\tau} r_{i j}(s)\left[f_{j}\left(y_{j}(t-s)\right)-f_{j}\left(y_{j}^{*}\right)\right] d s\right\} \\
& +\sum_{j=1}^{m} \operatorname{sgn}\left(v_{j}(t)\right)\left\{-c_{j} v_{j}(t)\right. \\
& +\sum_{i=1}^{n} d_{j i}\left[g_{i}\left(x_{i}(t)\right)-g_{i}\left(x_{i}^{*}\right)\right] \\
& +\int_{0}^{\tau}\left[\left|v_{j}(t)\right|-\left|v_{j}(t-s)\right|\right] d s+\sum_{j=1}^{\tau} \sum_{i=1}^{n} G_{i} P_{j i} \\
& \left.+\sum_{i=1}^{\tau} \int_{0}^{n} p_{j i}(s)\left[g_{i} x_{i}(t-s)-g_{i}\left(x_{i}^{*}\right)\right] d s\right\} \\
& +\sum_{i=1}^{n} \sum_{j=1}^{m} F_{j}\left|b_{i j}\right|\left[\left|v_{j}(t)\right|-\left|v_{j}(t-\tau)\right|\right] \\
& +\sum_{j=1}^{m} \sum_{i=1}^{n} G_{i}\left|d_{j i}\right|\left[\left|u_{i}(t)\right|-\left|u_{i}(t-\tau)\right|\right]+\sum_{i=1}^{n} \sum_{j=1}^{m} F_{j} R_{i j}
\end{aligned}
$$

By computations, we have

$$
\begin{aligned}
\frac{d^{+} V(t)}{d t} \leqslant & -\sum_{i=1}^{n} a_{i}\left|u_{i}(t)\right|-\sum_{j=m}^{n} c_{j}\left|v_{j}(t)\right| \\
& +\sum_{i=1}^{n} \sum_{j=1}^{m} F_{j}\left|b_{i j}\right|\left|v_{j}(t-\tau)\right| \\
& +\sum_{j=1}^{m} \sum_{i=1}^{n} G_{i}\left|d_{j i}\right|\left|u_{i}(t-\tau)\right|
\end{aligned}
$$

$+\sum_{i=1}^{n} \sum_{j=1}^{m} F_{j} R_{i j} \int_{0}^{\tau}\left|v_{j}(t-s)\right| d s$

$+\sum_{j=1}^{m} \sum_{i=1}^{n} G_{i} P_{j i} \int_{0}^{\tau}\left|u_{i}(t-s)\right| d s$

$+\sum_{i=1}^{n} \sum_{j=1}^{m} F_{j}\left|b_{i j}\right|\left[\left|v_{j}(t)\right|-\left|v_{j}(t-\tau)\right|\right]$

$+\sum_{j=1}^{m} \sum_{i=1}^{n} G_{i}\left|d_{j i}\right|\left[\left|u_{i}(t)\right|-\left|u_{i}(t-\tau)\right|\right]$

$+\sum_{i=1}^{n} \sum_{j=1}^{m} F_{j} R_{i j} \int_{0}^{\tau}\left[\left|v_{j}(t)\right|-\left|v_{j}(t-s)\right|\right] d s$

$+\sum_{j=1}^{m} \sum_{i=1}^{n} G_{i} P_{j i} \int_{0}^{\tau}\left[\left|u_{i}(t)\right|-\left|u_{i}(t-s)\right|\right] d s$

$\leqslant-\sum_{i=1}^{n} a_{i}\left|u_{i}(t)\right|-\sum_{j=m}^{n} c_{j}\left|v_{j}(t)\right|$

$+\sum_{i=1}^{n} \sum_{j=1}^{m} F_{j}\left|b_{i j}\right|\left|v_{j}(t)\right|$

$+\sum_{j=1}^{m} \sum_{i=1}^{n} G_{i}\left|d_{j i}\right|\left|u_{i}(t)\right|$

$+\sum_{i=1}^{n} \sum_{j=1}^{m} F_{j} R_{i j} \int_{0}^{\tau}\left|v_{j}(t)\right| d s$

$+\sum_{j=1}^{m} \sum_{i=1}^{n} G_{i} P_{j i} \int_{0}^{\tau}\left|u_{i}(t)\right| d s$

$\leqslant \sum_{i=1}^{n}\left\{-a_{i}+G_{i} \sum_{j=1}^{m}\left(\left|d_{j i}\right|+\tau P_{j i}\right)\right\}\left|u_{i}(t)\right|$

$+\sum_{j=1}^{m}\left\{-c_{j}+F_{j} \sum_{i=1}^{n}\left(\left|b_{i j}\right|+\tau R_{i j}\right)\right\}\left|v_{j}(t)\right|$

$\leqslant-\frac{\varepsilon}{\Gamma(1-\alpha)} \sum_{i=1}^{n}\left|u_{i}(t)\right|$

$-\frac{\varepsilon}{\Gamma(1-\beta)} \sum_{j=1}^{m}\left|v_{j}(t)\right|, \quad t \neq t_{k}$,

which implies that $d^{+} V(t) / d t \leqslant 0$ as $t \neq t_{k}$. Hence, for any $t \in\left[t_{k-1}, t_{k}\right)$, we get

$$
\begin{aligned}
& V(t)+\int_{t_{k-1}}^{t_{k}}\left[\frac{\varepsilon}{\Gamma(1-\alpha)} \sum_{i=1}^{n}\left|u_{i}(s)\right|\right. \\
& \left.+\frac{\varepsilon}{\Gamma(1-\beta)} \sum_{j=1}^{m}\left|v_{j}(s)\right|\right] d s \leqslant V\left(t_{k-1}^{+}\right) .
\end{aligned}
$$


Case 2. For $t=t_{k}$, from (29), one has

$$
\begin{aligned}
V\left(t_{k}^{+}\right)= & { }_{0} D_{t_{k}^{+}}^{-(1-\alpha)}\left[\sum_{i=1}^{n}\left|u_{i}\left(t_{k}^{+}\right)\right|\right] \\
& +{ }_{0} D_{t_{k}^{+}}^{-(1-\beta)}\left[\sum_{j=1}^{m}\left|v_{j}\left(t_{k}^{+}\right)\right|\right] \\
& +\sum_{i=1}^{n} \sum_{j=1}^{m} F_{j}\left|b_{i j}\right| \int_{t_{k}^{+}-\tau}^{t_{k}^{+}}\left|v_{j}(s)\right| d s \\
& +\sum_{j=1}^{m} \sum_{i=1}^{n} G_{i}\left|d_{j i}\right| \int_{t_{k}^{+}-\tau}^{t_{k}^{+}}\left|u_{i}(s)\right| d s \\
& +\sum_{i=1}^{n} \sum_{j=1}^{m} F_{j} R_{i j} \int_{0}^{\tau} \int_{t_{k}^{+}-s}^{t_{k}^{+}}\left|v_{j}(\eta)\right| d \eta d s \\
& +\sum_{j=1}^{m} \sum_{i=1}^{n} G_{i} P_{j i} \int_{0}^{\tau} \int_{t_{k}^{+}-s}^{t_{k}^{+}}\left|u_{i}(\eta)\right| d \eta d s .
\end{aligned}
$$

From (H3), we get

$$
\begin{aligned}
V\left(t_{k}^{+}\right)= & { }_{0} D_{t_{k}^{+}}^{-(1-\alpha)}\left[\sum_{i=1}^{n}\left|1-\lambda_{i k}^{(1)}\right|\left|u_{i}\left(t_{k}^{-}\right)\right|\right] \\
& +{ }_{0} D_{t_{k}^{+}}^{-(1-\beta)}\left[\sum_{j=1}^{m}\left|1-\lambda_{j k}^{(2)}\right|\left|v_{j}\left(t_{k}^{-}\right)\right|\right] \\
& +\sum_{i=1}^{n} \sum_{j=1}^{m} F_{j}\left|b_{i j}\right| \int_{t_{k}^{+}-\tau}^{t_{k}^{+}}\left|v_{j}(s)\right| d s \\
& +\sum_{j=1}^{m} \sum_{i=1}^{n} G_{i}\left|d_{j i}\right| \int_{t_{k}^{+}-\tau}^{t_{k}^{+}}\left|u_{\mathrm{i}}(s)\right| d s \\
& +\sum_{i=1}^{n} \sum_{j=1}^{m} F_{j} R_{i j} \int_{0}^{\tau} \int_{t_{k}^{+}-s}^{t_{k}^{+}}\left|v_{j}(\eta)\right| d \eta d s \\
& +\sum_{j=1}^{m} \sum_{i=1}^{n} G_{i} P_{j i} \int_{0}^{\tau} \int_{t_{k}^{+}-s}^{t_{k}^{+}}\left|u_{i}(\eta)\right| d \eta d s .
\end{aligned}
$$

Note that the inequalities $\left|1-\lambda_{i k}^{(1)}\right|<1$ and $\left|1-\lambda_{j k}^{(2)}\right|<1$ hold; then

$$
\begin{aligned}
V\left(t_{k}^{+}\right) \leqslant & { }_{0} D_{t_{k}^{-}}^{-(1-\alpha)}\left[\sum_{i=1}^{n}\left|u_{i}\left(t_{k}^{-}\right)\right|\right] \\
& +{ }_{0} D_{t_{k}^{-}}^{-(1-\beta)}\left[\sum_{j=1}^{m}\left|v_{j}\left(t_{k}^{-}\right)\right|\right] \\
& +\sum_{i=1}^{n} \sum_{j=1}^{m} F_{j}\left|b_{i j}\right| \int_{t_{k}^{+}-\tau}^{t_{k}^{+}}\left|v_{j}(s)\right| d s \\
& +\sum_{j=1}^{m} \sum_{i=1}^{n} G_{i}\left|d_{j i}\right| \int_{t_{k}^{+}-\tau}^{t_{k}^{+}}\left|u_{i}(s)\right| d s
\end{aligned}
$$

$$
\begin{aligned}
& \quad+\sum_{i=1}^{n} \sum_{j=1}^{m} F_{j} R_{i j} \int_{0}^{\tau} \int_{t_{k}^{+}-s}^{t_{k}^{+}}\left|v_{j}(\eta)\right| d \eta d s \\
& +\sum_{j=1}^{m} \sum_{i=1}^{n} G_{i} P_{j i} \int_{0}^{\tau} \int_{t_{k}^{+}-s}^{t_{k}^{+}}\left|u_{i}(\eta)\right| d \eta d s=V\left(t_{k}^{-}\right) \\
& =V\left(t_{k}\right) .
\end{aligned}
$$

Let $U(t)=\sum_{i=1}^{n}\left|u_{i}(t)\right|+\sum_{j=1}^{m}\left|v_{j}(t)\right|$, for any $t \in\left[t_{k-1}, t_{k}\right)$; then we have the following estimations:

$$
\begin{aligned}
V(t) & \leqslant-\int_{t_{k-1}}^{t} U(s) d s+V\left(t_{k-1}^{+}\right) \\
& \leqslant-\int_{t_{k-1}}^{t} U(s) d s+V\left(t_{k-1}^{-}\right) \\
& \leqslant-\int_{t_{k-2}}^{t} U(s) d s+V\left(t_{k-2}^{-}\right) \leqslant \cdots \\
& \leqslant-\int_{0}^{t} U(s) d s+V(0)
\end{aligned}
$$

Thus, we can get the following inequality:

$$
V(t)+\int_{0}^{t} U(s) d s \leqslant V(0),
$$

which implies that $\lim _{t \rightarrow+\infty} U(t)$ is bounded. From (28), $\left|{ }_{0}^{\mathrm{RL}} D_{t}^{\alpha} u_{i}(t)\right|$ and $\left|{ }_{0}^{\mathrm{RL}} D_{t}^{\beta} v_{j}(t)\right|$ are also bounded. From Lemma 5, we have $\lim _{t \rightarrow+\infty} \sum_{i=1}^{n}\left|u_{i}(t)\right|=0$ and $\lim _{t \rightarrow+\infty} \sum_{j=1}^{m}\left|v_{j}(t)\right|=$ 0 . Therefore, according to Lyapunov stability theory, a unique equilibrium solution $\left(x^{* T}, y^{* T}\right)^{T}$ for system (6) is globally asymptotically stable. This completes the proof.

The following corollary is the direct result of Theorem 11.

Corollary 12. Suppose that (H1)-(H3) hold; then a unique equilibrium solution for system (6) is globally asymptotically stable, if the following inequalities simultaneously hold for a small enough constant $\varepsilon>0$

$$
\begin{aligned}
\omega_{1} & =\max _{1 \leqslant i \leqslant n}\left\{\frac{\varepsilon}{\Gamma(1-\alpha)} \cdot \frac{1}{a_{i}}+\frac{G_{i}}{a_{i}} \sum_{j=1}^{m}\left[\left|d_{j i}\right|+\tau P_{j i}\right]\right\} \\
& <1, \\
\omega_{2} & =\max _{1 \leqslant j \leqslant m}\left\{\frac{\varepsilon}{\Gamma(1-\beta)} \cdot \frac{1}{c_{j}}+\frac{F_{j}}{c_{j}} \sum_{i=1}^{n}\left[\left|b_{i j}\right|+\tau R_{i j}\right]\right\} \\
& <1 .
\end{aligned}
$$

Remark 13. Different from fractional Lyapunov functional method in [30, 32, 37], an appropriate Lyapunov functional composed of fractional integral and definite integral terms in the proof of Theorem 11 is presented, and we only 
need to calculate its first-order derivative to derive stability conditions. As discussed in [35], in general speaking, it is very difficult to calculate the fractional-order derivatives of a Lyapunov functional. The main advantage of our constructed method is that we can avoid computing the fractional-order derivatives of the Lyapunov functional.

Remark 14 . The globally asymptotic stability criteria of a unique equilibrium solution for system (6) are described by the algebraic inequalities, which are dependent on the orders $\alpha$ and $\beta$ of fractional derivatives and reflect the close relation between the coefficients, neuron activation functions, and time-delay of network parameters. Moreover, the globally asymptotic stability criteria are more easily checked and contribute to reducing the computational burden.

Remark 15. When $\alpha=\beta=1$, system (6) is reduced to integerorder BAM neural networks with distributed delays and impulses [23]. Note that the Riemann-Liouville derivative is a continuous operator of the order (see [9-11]); then we can obtain globally asymptotic stability criteria for impulsive integer-order hybrid BAM neural networks from the proof of Theorem 11.

Remark 16. In [33, 34, 44], the authors have focused on studying the finite-time stability of fractional-order delayed neural networks. However, it should be pointed out that the finite-time stability and asymptotic stability in the sense of Lyapunov are different concepts, because finite-time stability does not contain Lyapunov asymptotic stability and vice versa $[34,47]$. This is also the motivation of this paper.

\section{An Illustrative Example}

In this section, an example for impulsive fractional-order hybrid BAM neural networks with distributed delays is given to illustrate the effectiveness and feasibility of the theoretical results.

Example 17. Consider the four-state Riemann-Liouville fractional-order hybrid BAM neural network model with distributed delays and impulsive effects described by

$$
\begin{aligned}
{ }_{0}^{\mathrm{RL}} D_{t}^{0.2} x_{1}(t)= & -0.7 x_{1}(t)-0.2 f_{1}\left(y_{1}(t)\right) \\
& +0.1 f_{2}\left(y_{2}(t)\right) \\
& +2 \int_{0}^{0.2} s f_{1}\left(y_{1}(t-s)\right) d s \\
& +\int_{0}^{0.2} s f_{2}\left(y_{2}(t-s)\right) d s, \\
{ }_{0}^{\mathrm{RL}} D_{t}^{0.2} x_{2}(t)= & -0.6 x_{2}(t)+0.3 f_{1}\left(y_{1}(t)\right) \\
& +0.2 f_{2}\left(y_{2}(t)\right) \\
& +\int_{0}^{0.2} s f_{1}\left(y_{1}(t-s)\right) d s \\
& -\int_{0}^{0.2} s^{3} f_{2}\left(y_{2}(t-s)\right) d s,
\end{aligned}
$$

$$
\begin{aligned}
\Delta x_{i}\left(t_{k}\right)= & -0.3\left(x_{i}\left(t_{k}\right)-x_{i}^{*}\right), \\
{ }_{0} D_{t}^{0.6} y_{1}(t)= & -0.7 y_{1}(t)+0.4 g_{1}\left(y_{1}(t)\right) \\
& +0.2 g_{2}\left(y_{2}(t)\right) \\
& -\int_{0}^{0.2} s g_{1}\left(y_{1}(t-s)\right) d s \\
& +\int_{0}^{0.2} s^{2} g_{2}\left(y_{2}(t-s)\right) d s, \\
{ }_{0}^{\mathrm{RL}} D_{t}^{0.6} y_{2}(t)= & -0.6 y_{2}(t)+0.1 g_{1}\left(y_{1}(t)\right) \\
& -0.3 g_{2}\left(y_{2}(t)\right) \\
& +\int_{0}^{0.2} s^{2} g_{1}\left(y_{1}(t-s)\right) d s \\
& +\int_{0}^{0.2} s g_{2}\left(y_{2}(t-s)\right) d s, \\
\Delta y_{j}\left(t_{k}\right)= & -0.4\left(y_{j}\left(t_{k}\right)-y_{i}^{*}\right), \\
& j=1,2 ; k=1,2, \ldots,
\end{aligned}
$$

where $\alpha=0.2, \beta=0.6, \tau=0.2, a_{1}=c_{1}=0.7, a_{2}=c_{2}=0.6$, $b_{11}=-0.2, b_{12}=0.1, b_{21}=0.3, b_{22}=0.2, d_{11}=0.4, d_{12}=0.2$, $d_{21}=0.1, d_{22}=-0.3, r_{11}(s)=2 s, r_{12}(s)=s, r_{21}(s)=s$, $r_{22}(s)=-s^{3}, p_{11}(s)=-s, p_{12}(s)=s^{2}, p_{21}(s)=s^{2}, p_{22}(s)=s$, and

$$
\begin{gathered}
f_{j}\left(y_{j}\right)=\frac{1}{2}\left(\left|y_{j}+1\right|-\left|y_{j}-1\right|\right), \quad j=1,2, \\
g_{i}\left(x_{i}\right)=\frac{1}{2}\left(\left|x_{i}+1\right|-\left|x_{i}-1\right|\right), \quad i=1,2 .
\end{gathered}
$$

From (43), we know that $F_{1}=F_{2}=G_{1}=G_{2}=1$. Since $f_{1}(0)=f_{2}(0)=0, g_{1}(0)=g_{2}(0)=0$, then $\left(x_{1}^{*}, x_{2}^{*}, y_{1}^{*}, y_{1}^{*}\right)^{T}=$ $(0,0,0,0)^{T}$ is an equilibrium solution for system (42). Next, we apply Theorem 11 or Corollary 12 to check the uniqueness and global asymptotic stability of the equilibrium point for system (42).

In fact, by computations, one can get that $R_{11}=0.4, R_{12}=$ $R_{21}=0.2, R_{22}=0.008, P_{11}=P_{22}=0.2$, and $P_{12}=P_{21}=0.04$. Choosing a positive constant $\varepsilon=0.04>0$, then we can obtain

$$
\begin{aligned}
\eta_{1} & =\min _{1 \leqslant i \leqslant 2}\left\{a_{i}-G_{i} \sum_{j=1}^{2}\left[\left|d_{j i}\right|+\tau P_{j i}\right]\right\}=0.052 \\
> & \frac{\varepsilon}{\Gamma(1-\alpha)}=0.044 \\
\eta_{2} & =\min _{1 \leqslant j \leqslant 2}\left\{c_{j}-F_{j} \sum_{i=1}^{2}\left[\left|b_{i j}\right|+\tau R_{i j}\right]\right\}=0.116 \\
& >\frac{\varepsilon}{\Gamma(1-\beta)}=0.045
\end{aligned}
$$



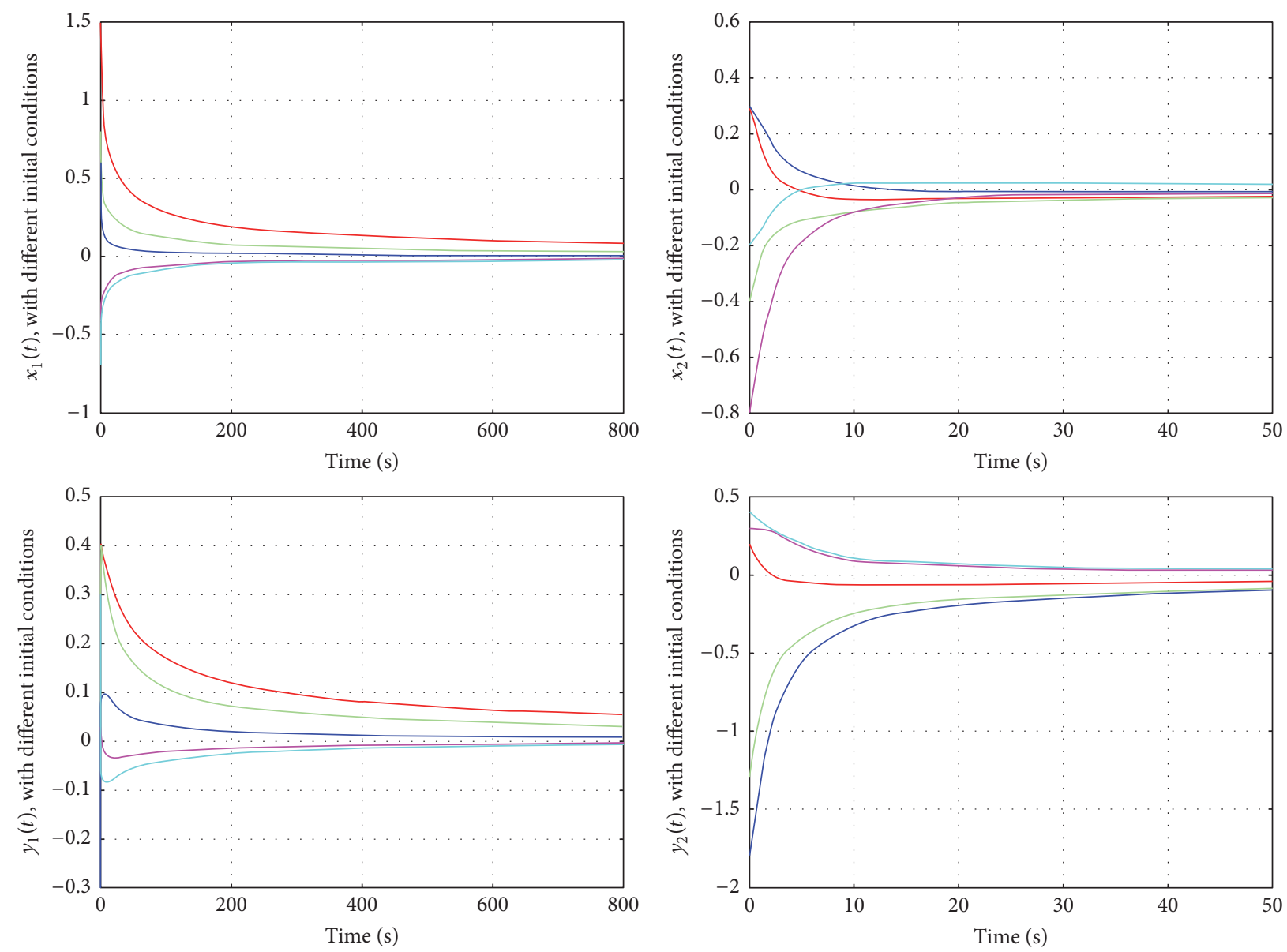

FIGURE 1: State trajectories of BAM neural network (42) with $\alpha=0.2 ; \beta=0.6$ under different initial conditions.

$$
\begin{aligned}
\omega_{1} & =\max _{1 \leqslant i \leqslant 2}\left\{\frac{\varepsilon}{\Gamma(1-\alpha)} \cdot \frac{1}{a_{i}}+\frac{G_{i}}{a_{i}} \sum_{j=1}^{2}\left[\left|d_{j i}\right|+\tau P_{j i}\right]\right\} \\
& =0.856<1, \\
\omega_{2} & =\max _{1 \leqslant j \leqslant 2}\left\{\frac{\varepsilon}{\Gamma(1-\beta)} \cdot \frac{1}{c_{j}}+\frac{F_{j}}{c_{j}} \sum_{i=1}^{2}\left[\left|b_{i j}\right|+\tau R_{i j}\right]\right\} \\
& =0.722<1 .
\end{aligned}
$$

Thus, the conditions of Theorem 11 or Corollary 12 are satisfied. For numerical simulations, Figure 1 depicts the state trajectories of system (42) under different initial conditions with $\alpha=0.2, \beta=0.6$. It can be directly observed that the unique equilibrium solution $(0,0,0,0)^{T}$ for system (42) is globally asymptotically stable with $\alpha=0.2, \beta=0.6$. Therefore, the numerical simulations further confirm the theoretical results of this paper.

\section{Conclusions}

In this paper, the sufficient conditions for the existence and uniqueness of the equilibrium solution are presented based

on the contraction mapping principle. By constructing a suitable Lyapunov functional composed of fractional integral and definite integral terms, we calculate its first-order derivative to derive global asymptotic stability of the equilibrium point. The constructed method avoids calculating the fractionalorder derivative of the Lyapunov functional. Furthermore, the presented results are described as the algebraic inequalities, which are convenient and feasible to verify the existence and asymptotic stability of the equilibrium solution. For further research, it is interesting and challenging to discuss the chaos phenomena, Hopf bifurcation, and synchronization control problem for fractional-order memristor-based hybrid BAM neural networks with leakage, time-varying, and distributed delays.

\section{Conflicts of Interest}

The authors declare that there are no conflicts of interest with regard to the publication of this paper.

\section{Acknowledgments}

This work is jointly supported by the National Natural Science Fund of China (11301308, 61573096, and 61272530), 
the 333 Engineering Fund of Jiangsu Province of China (BRA2015286), the Natural Science Fund of Anhui Province of China (1608085MA14), the Key Project of Natural Science Research of Anhui Higher Education Institutions of China (gxyqZD2016205 and KJ2015A152), and the Natural Science Youth Fund of Jiangsu Province of China (BK20160660).

\section{References}

[1] R. Metzler and J. Klafter, "The random walk's guide to anomalous diffusion: a fractional dynamics approach," Physics Reports. A Review Section of Physics Letters, vol. 339, no. 1, pp. 1-77, 2000.

[2] R. Hilfer, Applications of Fractional Calculus in Physics, World Scientific, Toh Tuck Link, Singapore, 2000.

[3] N. Laskin, "Fractional market dynamics," Physica A. Statistical Mechanics and its Applications, vol. 287, no. 3-4, pp. 482-492, 2000.

[4] J. Sabatier, O. P. Agrawal, and J. A. Tenreiro Machado, Advances in Fractional Calculus: Theoretical Developments and Applications in Physics and Engineering, Springer, Dordrecht, Netherlands, 2007.

[5] D. Baleanu, J. A. Tenreiro Machado, and A. C. J. Luo, Fractional Dynamics and Control, Springer, New York, NY, USA, 2012.

[6] R. L. Magin, "Fractional calculus models of complex dynamics in biological tissues," Computers \& Mathematics with Applications, vol. 59, no. 5, pp. 1586-1593, 2010.

[7] W. C. Chen, "Nonlinear dynamics and chaos in a fractionalorder financial system," Chaos, Solitons and Fractals, vol. 36, no. 5, pp. 1305-1314, 2008.

[8] E. Ahmed and A. S. Elgazzar, "On fractional order differential equations model for nonlocal epidemics," Physica A: Statistical Mechanics and Its Applications, vol. 379, no. 2, pp. 607-614, 2007.

[9] I. Podlubny, Fractional Differential Equations, vol. 198 of Mathematics in Science and Engineering, Academic Press, San Diego, Calif, USA, 1999.

[10] A. A. Kilbas, H. M. Srivastava, and J. J. Trujillo, Theory and Applications of Fractional Differential Equations, New York, NY, USA, Elsevier, 2006.

[11] K. Diethelm, The Analysis of Fractional Differential Equations, vol. 2004 of Lecture Notes in Mathematics, Springer, Berlin, Germany, 2010.

[12] B. Xin, T. Chen, and Y. Liu, "Projective synchronization of chaotic fractional-order energy resources demand-supply systems via linear control," Communications in Nonlinear Science and Numerical Simulation, vol. 16, no. 11, pp. 4479-4486, 2011.

[13] A. E. Matouk, "Chaos synchronization of a fractional-order modified van der Pol-Duffing system via new linear control, backstepping control and Takagi-Sugeno fuzzy approaches," Complexity, vol. 21, no. S1, pp. 116-124, 2016.

[14] A. Y. Leung, X.-F. Li, Y.-D. Chu, and X.-B. Rao, "Synchronization of fractional-order chaotic systems using unidirectional adaptive full-state linear error feedback coupling," Nonlinear Dynamics, vol. 82, no. 1-2, pp. 185-199, 2015.

[15] A. M. El-Sayed, H. M. Nour, A. Elsaid, A. E. Matouk, and A. Elsonbaty, "Dynamical behaviors, circuit realization, chaos control, and synchronization of a new fractional order hyperchaotic system," Applied Mathematical Modelling. Simulation and Computation for Engineering and Environmental Systems, vol. 40, no. 5-6, pp. 3516-3534, 2016.
[16] A. M. El-Sayed, A. Elsonbaty, A. A. Elsadany, and A. E. Matouk, "Dynamical analysis and circuit simulation of a new fractionalorder hyperchaotic system and its discretization," International Journal of Bifurcation and Chaos in Applied Sciences and Engineering, vol. 26, no. 13, Article ID 1650222, pp. 1-35, 2016.

[17] J. Cao, "Global exponential stability of hopfield neural networks," International Journal of Systems Science, vol. 32, no. 2, pp. 233-236, 2001.

[18] S. Dharani, R. Rakkiyappan, and J. Cao, "New delay-dependent stability criteria for switched Hopfield neural networks of neutral type with additive time-varying delay components," Neurocomputing, vol. 151, no. 2, pp. 827-834, 2015.

[19] J. Cao, D.-S. Huang, and Y. Qu, "Global robust stability of delayed recurrent neural networks," Chaos, Solitons \& Fractals, vol. 23, no. 1, pp. 221-229, 2005.

[20] Y. Liu, Z. Wang, and X. Liu, "Global exponential stability of generalized recurrent neural networks with discrete and distributed delays," Neural Networks, vol. 19, no. 5, pp. 667-675, 2006.

[21] Q. Zhang, X. Wei, and J. Xu, "Stability of delayed cellular neural networks," Chaos, Solitons \& Fractals, vol. 31, no. 2, pp. 514-520, 2007.

[22] W. Xiong, Y. Shi, and J. Cao, "Stability analysis of twodimensional neutral-type Cohen-Grossberg BAM neural networks," Neural Computing and Applications, vol. 28, no. 4, pp. 703-716, 2017.

[23] Z.-T. Huang, X.-S. Luo, and Q.-G. Yang, "Global asymptotic stability analysis of bidirectional associative memory neural networks with distributed delays and impulse," Chaos, Solitons \& Fractals, vol. 34, no. 3, pp. 878-885, 2007.

[24] J. H. Park, C. H. Park, O. M. Kwon, and S. M. Lee, "A new stability criterion for bidirectional associative memory neural networks of neutral-type," Applied Mathematics and Computation, vol. 199, no. 2, pp. 716-722, 2008.

[25] Y. Li and S. Gao, "Global exponential stability for impulsive BAM neural networks with distributed delays on time scales," Neural Processing Letters, vol. 31, no. 1, pp. 65-91, 2010.

[26] C. Song and J. Cao, "Dynamics in fractional-order neural networks," Neurocomputing, vol. 142, pp. 494-498, 2014.

[27] H.-B. Bao and J.-D. Cao, "Projective synchronization of fractional-order memristor-based neural networks," Neural Networks, vol. 63, pp. 1-9, 2015.

[28] F. Ren, F. Cao, and J. Cao, "Mittag-Leffler stability and generalized Mittag-Leffler stability of fractional-order gene regulatory networks," Neurocomputing, vol. 160, pp. 185-190, 2015.

[29] X. Yang, C. Li, Q. Song, T. Huang, and X. Chen, "Mittag-Leffler stability analysis on variable-time impulsive fractional-order neural networks," Neurocomputing, vol. 207, pp. 276-286, 2015.

[30] Y. Li, Y. Chen, and I. Podlubny, "Stability of fractional-order nonlinear dynamic systems: Lyapunov direct method and generalized Mittag-Leffler stability," Computers \& Mathematics with Applications, vol. 59, no. 5, pp. 1810-1821, 2010.

[31] H. Bao, J. H. Park, and J. Cao, "Adaptive synchronization of fractional-order memristor-based neural networks with time delay," Nonlinear Dynamics. An International Journal of Nonlinear Dynamics and Chaos in Engineering Systems, vol. 82, no. 3, pp. 1343-1354, 2015.

[32] I. Stamova, "Global Mittag-Leffler stability and synchronization of impulsive fractional-order neural networks with timevarying delays," Nonlinear Dynamics. An International Journal of Nonlinear Dynamics and Chaos in Engineering Systems, vol. 77, no. 4, pp. 1251-1260, 2014. 
[33] Y. Ke and C. Miao, "Stability analysis of fractional-order CohenGrossberg neural networks with time delay," International Journal of Computer Mathematics, vol. 92, no. 6, pp. 1102-1113, 2015.

[34] X. Ding, J. Cao, X. Zhao, and F. E. Alsaadi, "Finite-time stability of fractional-order complex-valued neural networks with time delays," Neural Processing Letters, pp. 1-20, 2017.

[35] H. Zhang, R. Ye, J. Cao, and A. Alsaedi, "Delay-independent stability of Riemann-Liouville fractional neutral-type delayed neural networks," Neural Processing Letters, pp. 1-16, 2017.

[36] R. Rakkiyappan, J. Cao, and G. Velmurugan, "Existence and uniform stability analysis of fractional-order complex-valued neural networks with time delays," IEEE Transactions on Neural Networks and Learning Systems, vol. 26, no. 1, pp. 84-97, 2015.

[37] R. Rakkiyappan, R. Sivaranjani, G. Velmurugan, and J. Cao, "Analysis of global $\mathrm{O}(\mathrm{t}-\alpha)$ stability and global asymptotical periodicity for a class of fractional-order complex-valued neural networks with time varying delays," Neural Networks, vol. 77, pp. 51-69, 2016.

[38] D. Matignon, "Stability results for fractional differential equations with applications to control processing," Computational Engineering in Systems Applications, vol. 2, pp. 963-968, 1996.

[39] A. E. Matouk, "Stability conditions, hyperchaos and control in a novel fractional order hyperchaotic system," Physics Letters A: General, Atomic and Solid State Physics, vol. 373, no. 25, pp. 2166-2173, 2009.

[40] D. Bainov and P. Simeonov, Impulsive Differential Equations: Periodic Solutions and Applications, John Wiley and Sons, New York, NY, USA, 1993.

[41] M. Benchohra, J. Henderson, and S. Ntouyas, Impulsive Differential Equations and Inclusions, vol. 2 of Contemporary Mathematics and Its Applications, Hindawi Publishing Corporation, New York, NY, USA, 2006.

[42] F. Wang, Y. Yang, and M. Hu, "Asymptotic stability of delayed fractional-order neural networks with impulsive effects," Neurocomputing, vol. 154, pp. 239-244, 2015.

[43] B. Kosko, "Adaptive bidirectional associative memories," Applied Optics, vol. 26, no. 23, pp. 4947-4960, 1987.

[44] C. Rajivganthi, F. A. Rihan, S. Lakshmanan, and P. Muthukumar, "Finite-time stability analysis for fractional-order CohenGrossberg BAM neural networks with time delays," Neural Computing and Applications, pp. 1-12, 2016.

[45] J. Xiao, S. Zhong, Y. Li, and F. Xu, "Finite-time Mittag-Leffler synchronization of fractional-order memristive BAM neural networks with time delays," Neurocomputing, vol. 219, pp. 431439, 2017.

[46] F. Wang, Y. Yang, X. Xu, and L. Li, "Global asymptotic stability of impulsive fractional-order BAM neural networks with time delay," Neural Computing and Applications, vol. 28, no. 2, pp. 345-352, 2017.

[47] L. V. Hien, "An explicit criterion for finite-time stability of linear nonautonomous systems with delays," Applied Mathematics Letters. An International Journal of Rapid Publication, vol. 30, pp. 12-18, 2014.

[48] A. Granas and J. Dugundji, Fixed Point Theory, Springer, New York, NY, USA, 2003. 


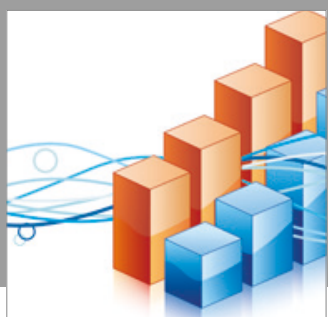

Advances in

Operations Research

vatersals

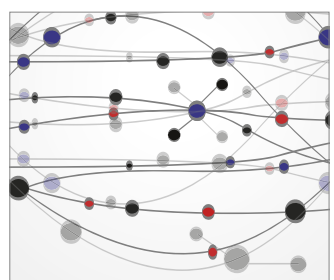

\section{The Scientific} World Journal
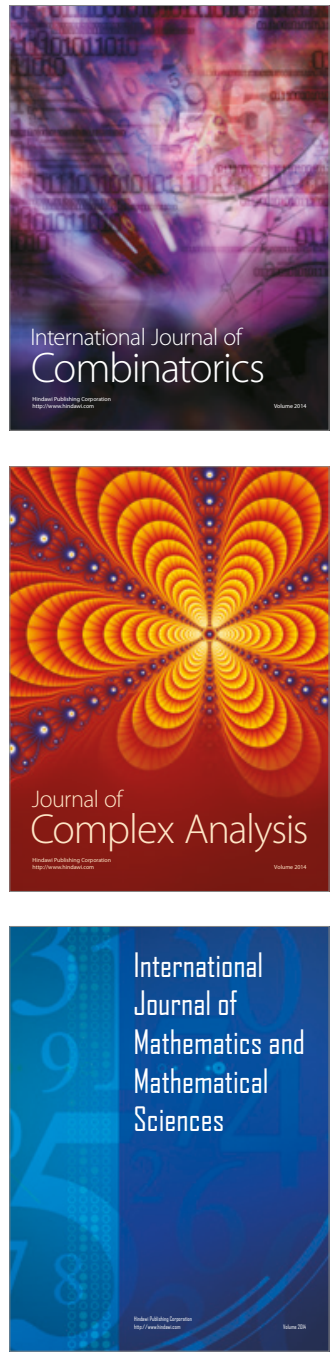
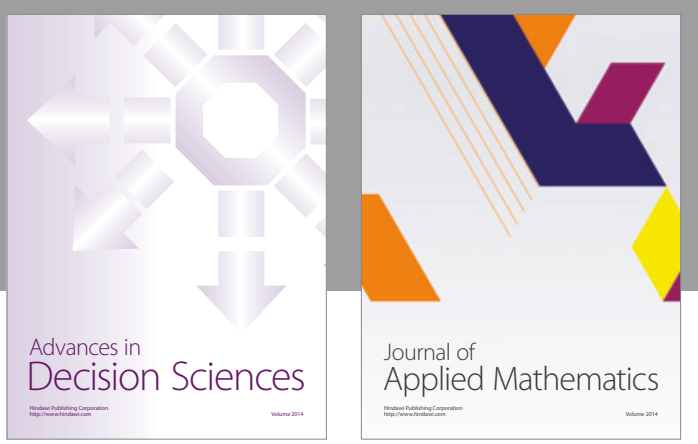

Algebra

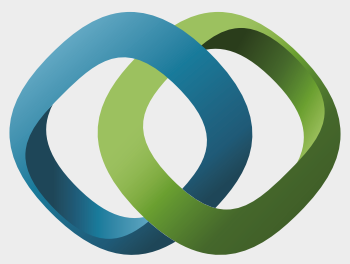

\section{Hindawi}

Submit your manuscripts at

https://www.hindawi.com
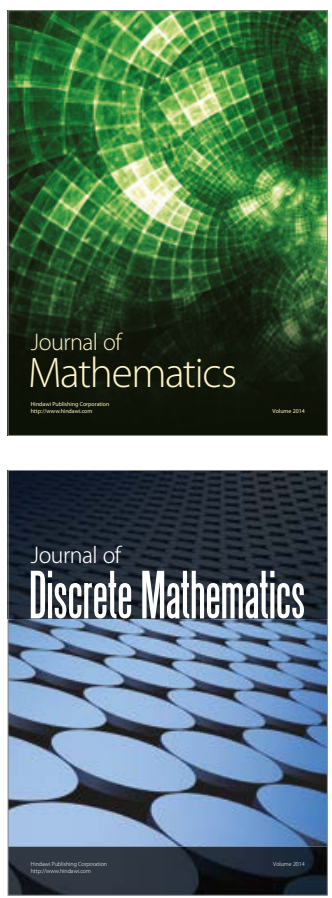

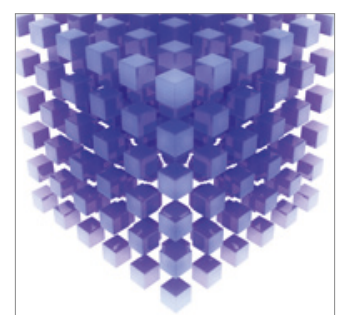

Mathematical Problems in Engineering
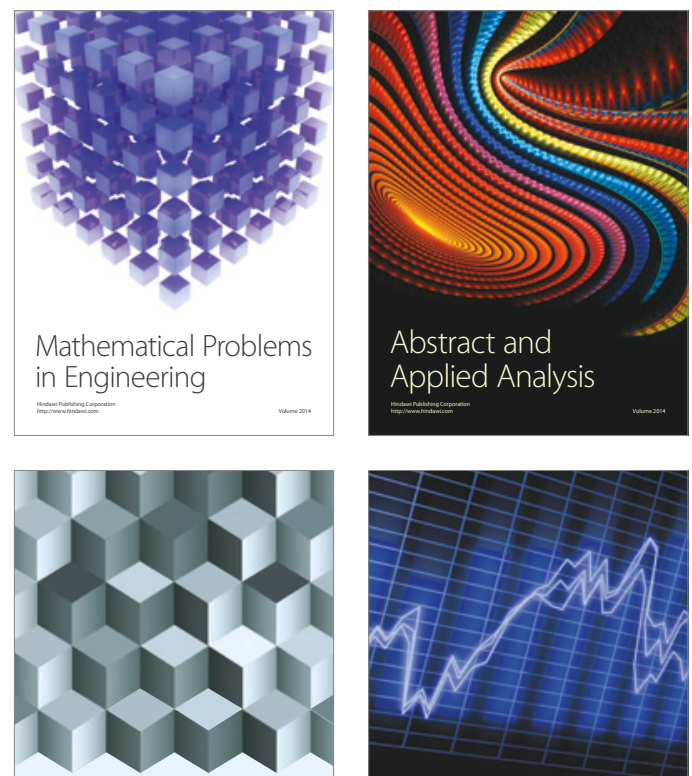

Journal of

Function Spaces

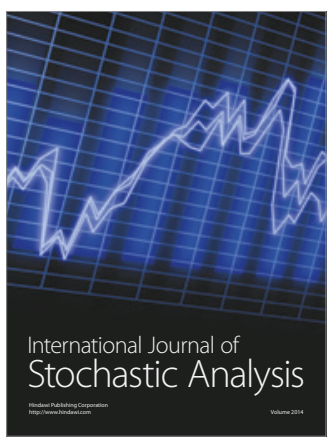

Probability and Statistics
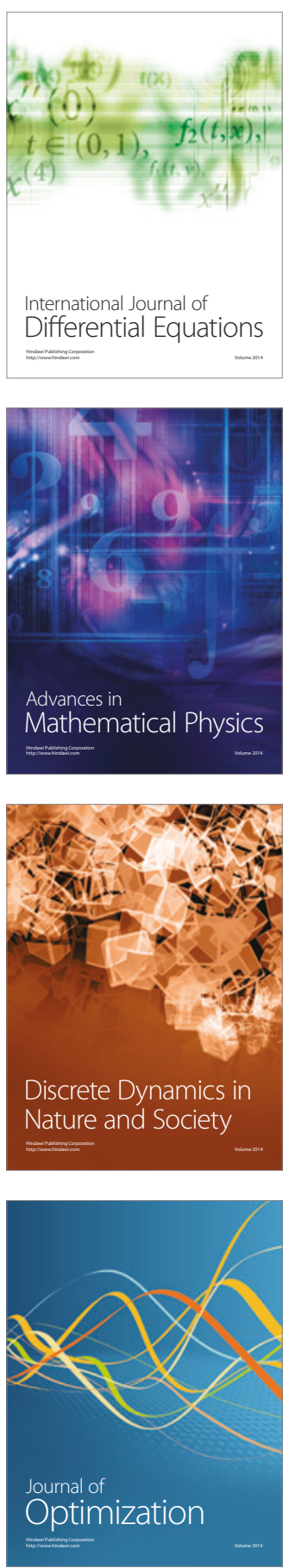